\title{
GㄷNTIEC
}

\section{AVALIAÇÃO DA DEGRADAÇÃO TÉRMICA E OXIDATIVA DE UM FOSFATO ÉSTER E DE UM ÓLEO NAFTÊNICO}

\section{EVALUATION OF THERMAL AND OXIDATIVE DEGRADATION OF A PHOSPHATE ESTER AND OF A NAPHTHENIC OIL}

\author{
Francisco Samuel da Cunha Lima Batista ${ }^{1}$; Maria Dalva Silva Fonseca ${ }^{1}$; Maria Alexsandra de Sousa Rios ${ }^{2}$ \\ ${ }^{1}$ Departamento de Química - DQ \\ Universidade Federal do Piauí - UFPI - Teresina/SE - Brasil \\ samuel.grintequi@gmail.com \\ dalva.grintequi@gmail.com \\ ${ }^{2}$ Departamento de Engenharia Mecânica - DEM \\ Universidade Federal do Ceará - UFC - Fortaleza/CE - Brasil \\ alexsandrarios@ufc.br
}

\begin{abstract}
Resumo
O presente trabalho apresenta o estudo da degradação térmica e oxidativa do fluido tritolil fosfato, bem como de um destilado leve de petróleo naftênico hidrotratado (óleo naftênico), através das técnicas de Espectroscopia no Infravermelho (FTIR), Análise Térmica (TG-DTG) e de um Teste de Oxidação Acelerada (TOA) desenvolvido em nosso laboratório. O monitoramento do processo de degradação foi realizado através de FTIR. De acordo com os resultados de TG-DTG observou-se que o tritolil fosfato possui uma boa estabilidade térmica e oxidativa compatível com os resultados para fosfatos ésteres. Estes compostos possuem uma boa estabilidade térmica devido à forte ligação P-O-Caromático. Quanto ao óleo naftênico, o mesmo não apresentou significativa estabilidade térmica iniciando suas etapas de oxidação/degradação em torno de 78 e $84{ }^{\circ} \mathrm{C}$. Os resultados de degradação obtidos por TG-DTG corroboraram com os resultados dos Testes de Oxidação Acelerada dos sistemas de oxidação desenvolvidos no laboratório, possibilitando a validação destes resultados.
\end{abstract}

Palavras-chave: organofosforado; sistema de oxidação acelerada; TG-DTG.

\begin{abstract}
The present work shows the study of thermal and oxidative degradation of the fluid tritolyl phosphate as well as, of a light distillated hydrotreated naphthenic oil (naphthenic oil) by spectroscopy techniques Infrared (FTIR), Thermal Analysis (TG-DTG) and an Accelerated Oxidation Test (TOA) developed in our laboratory. The degradation process was monitored by FTIR. According to the results of TG-DTG was observed that the tritolyl phosphate possess a good thermal and oxidative stability compatible with results of phosphate esters. These compounds have a good thermal stability due to the strong bond PO-Caromatic. The naphthenic oil not showed a
\end{abstract}


significant thermal stability beginning its oxidation/degradation stage around 78 and $84{ }^{\circ} \mathrm{C}$. The degradation results of TG-DTG corroborate with the results of Accelerated Oxidation Test of the systems developed in the laboratory, making it possible to validate these results.

Key-words: organophosphorus; accelerated oxidation system; TG-DTG.

\subsection{Introdução}

Nos últimos anos a indústria petroquímica tem se preocupado com a melhoria do desempenho de seus produtos (lubrificantes, graxas, gasolina e etc.). Neste contexto, tal setor tem incentivado pesquisas voltadas ao desenvolvimento/aperfeiçoamento de novos produtos de modo que possam atender a demanda industrial (Rios et al., 2005). Na linha de lubrificantes de alto desempenho, os fosfatos ésteres assumem posição de destaque uma vez que são muito utilizados como lubrificantes em sistemas hidráulicos e de aviação (Rios e Mazzetto, 2012; Rios e Mazzetto, 2012).

Dentre os derivados petroquímicos de grande demanda, os lubrificantes parafínicos e naftênicos também são bastante utilizados nos processos de lubrificação de veículos e máquinas em geral, apresentando grande importância para o setor de derivados do petróleo (Rios et al., 2007). Uma vez que as máquinas industriais são compostas por peças metálicas; e estas se desgastam com o tempo em decorrência do funcionamento, o setor de química fina tem cada vez mais investido em novas moléculas multifuncionais, as quais, quando incorporadas aos substratos orgânicos melhoram sobremaneira suas principais propriedades (Santos et al., 2001; Rios et al., 2010; Howwel and AlOmari, 2012).

Conforme citado anteriormente, os lubrificantes sejam sintéticos, semissintéticos ou derivados da biomassa são bastante aplicados nas indústrias de automóvel (Façanha, 2008; Alves, 2010), de aviões e equipamentos pesados, os quais por serem submetidos a condições extremas se deterioram total ou parcialmente, formando compostos oxigenados, compostos aromáticos polinucleares de alta viscosidade, resinas e lacas (Rios et al., 2010). Como a viscosidade do lubrificante é uma propriedade que deve ser mantida dentro dos limites padrão, a alteração desta pode ser utilizada como parâmetro de monitoramento para verificação da qualidade deste produto. Assim, a diminuição da viscosidade pode ser um indicativo de contaminação causada por solvente ou combustível; e o aumento da viscosidade pode representar um indicativo da presença de contaminantes insolúveis, oxidação acentuada e contaminação com água (Façanha, 2008).

Em superfícies metálicas, a função da parafina e dos óleos lubrificantes é suavizar o atrito, dissipar o calor e minimizar o desgaste entre as superfícies que se movem uma contra outra (Schumacher and Zinke, 1997). No que concerne à degradação, a oxidação é o principal agente de deterioração dos óleos lubrificantes durante o tempo de vida ou até mesmo no seu armazenamento (Rios et al., 2010). Deste modo, as indústrias químicas têm incorporado às formulações dos 
lubrificantes aditivos de alto desempenho, buscando um meio viável para controlar e/ou melhorar a estabilidade dos referidos produtos hidrocarbônicos.

Conforme dito anteriormente, dentre os lubrificantes comerciais aplicados industrialmente, os fosfatos ésteres são largamente utilizados como fluidos hidráulicos em aviação devido a sua boa estabilidade térmica (Exxon Mobil Corporation, 2008). Dentro deste contexto, no presente estudo térmico foi utilizado um fluido da classe dos organofosforados, o produto comercial tritolil fosfato (CAS: 1330-78-5); e um óleo naftênico hidrogenado (CAS: 64742-53-6). Segundo Schwetlick e colaboradores (1991), os fosfatos ésteres são compostos que interagem fortemente com a superfície metálica, evitando o contato metal-metal (Schumacher et al., 1991).

Outra característica bastante importante dos organofosforados é o fato de serem estáveis em condições extremas, bem como se apresentarem no estado líquido, facilitando a miscibilidade quando adicionados a formulações lubrificantes (Kirpichnikov et al., 1983).

Diante do exposto, o presente trabalho apresenta o estudo da degradação térmica e oxidativa do fluido tritolil fosfato, bem como de um destilado leve de petróleo naftênico hidrotratado (óleo naftênico), através das técnicas de Espectroscopia no Infravermelho (FTIR), Análise Térmica (TGDTG) e Teste de Oxidação Acelerada (TOA) (Neto et al., 2004).

\subsection{Materiais e Métodos}

\section{Materiais}

O fosfato éster "tritolil fosfato" (CAS No. 1330-78-5) foi adquirido na Sigma Aldrich Chemical Company (grau analítico) e o destilado leve de petróleo naftênico hidrotratado (CAS No. 64742-53-6) foi gentilmente cedido pela Refinaria Lubrificantes e Derivados do Nordeste (Lubnor), localizada no município de Fortaleza, estado do Ceará, o qual foi utilizado sem nenhum tratamento prévio.

\section{Caracterizações}

A espectroscopia na região do infravermelho por transformada de Fourier (FTIR) foi realizada em um espectrômetro de Marca VARIAN, modelo 660-IR. Amostras foram coletadas e os espectros registrados a cada 24 horas. O comportamento térmico das amostras foi analisado em uma termobalança Shimadzu, Modelo TGA-50H, de $25{ }^{\circ} \mathrm{C}$ a $800{ }^{\circ} \mathrm{C}$, massa de amostra de aproximadamente $6 \mathrm{mg}$, a uma taxa de aquecimento de $10{ }^{\circ} \mathrm{C} \mathrm{min}^{-1}$, sob fluxo de ar sintético e

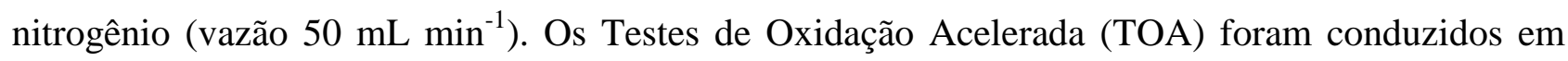
dois sistemas de oxidação desenvolvidos em nosso laboratório, ver Figuras 1 e 2. Para avaliação da 
degradação térmica e oxidativa do tritolil fosfato foram utilizados dois subsistemas (Figura 1), um com borbulhamento de ar a uma vazão de $0,5 \mathrm{~L} \mathrm{~min}^{-1} \mathrm{e}$ outro com agitação constante sem borbulhamento de ar, ambos submetidos ao aquecimento em um banho de óleo a uma temperatura de $150{ }^{\circ} \mathrm{C} \pm 5{ }^{\circ} \mathrm{C}$, por 96 horas. Para o monitoramento do processo de degradação do composto via FTIR, amostras de $0,5 \mathrm{~g}$ foram coletadas e os espectros registrados a cada 24 horas. Para a avaliação de degradação do óleo naftênico foram utilizados três subsistemas (Figura 2), com borbulhamento de $\operatorname{ar}\left(0,5 \mathrm{~L} \mathrm{~min}^{-1}\right)$ e aquecimento em banho de óleo a $150{ }^{\circ} \mathrm{C} \pm 5^{\circ} \mathrm{C}$, por 168 horas (ASTM D2440, 2013). O monitoramento do processo oxidativo foi realizado também por FTIR. Amostras foram coletas e os espectros foram registrados a cada 24 horas.

Figura 1. Sistema de oxidação utilizado para degradação do fosfato éster "tritolil fosfato".

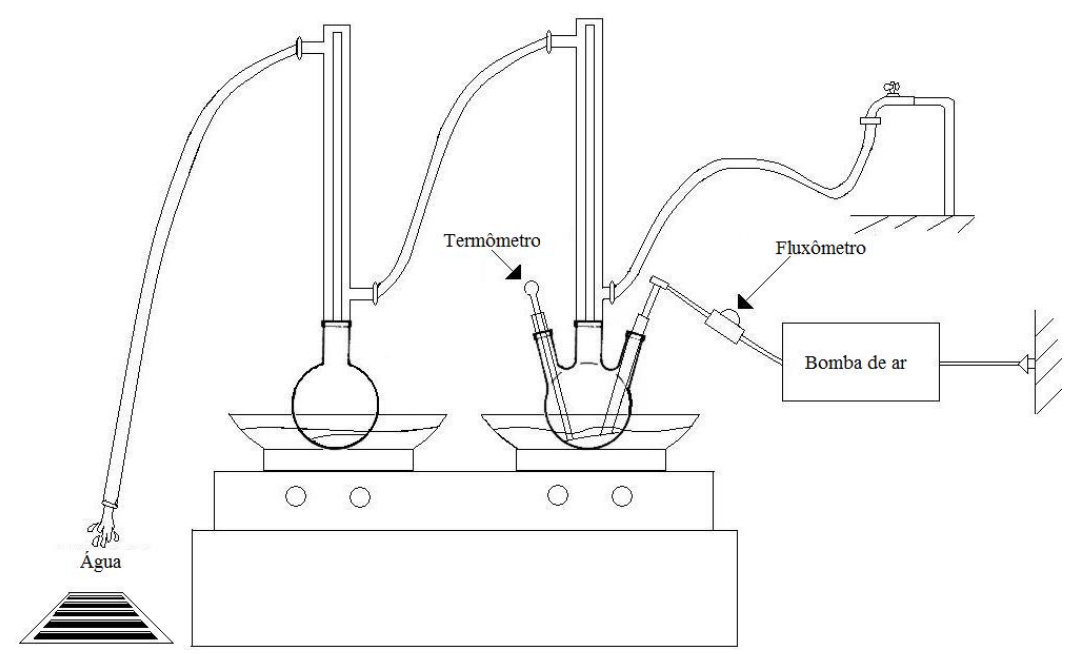

Fonte: Autoria própria.

Figura 2. Sistema de oxidação utilizado para degradação do óleo naftênico.

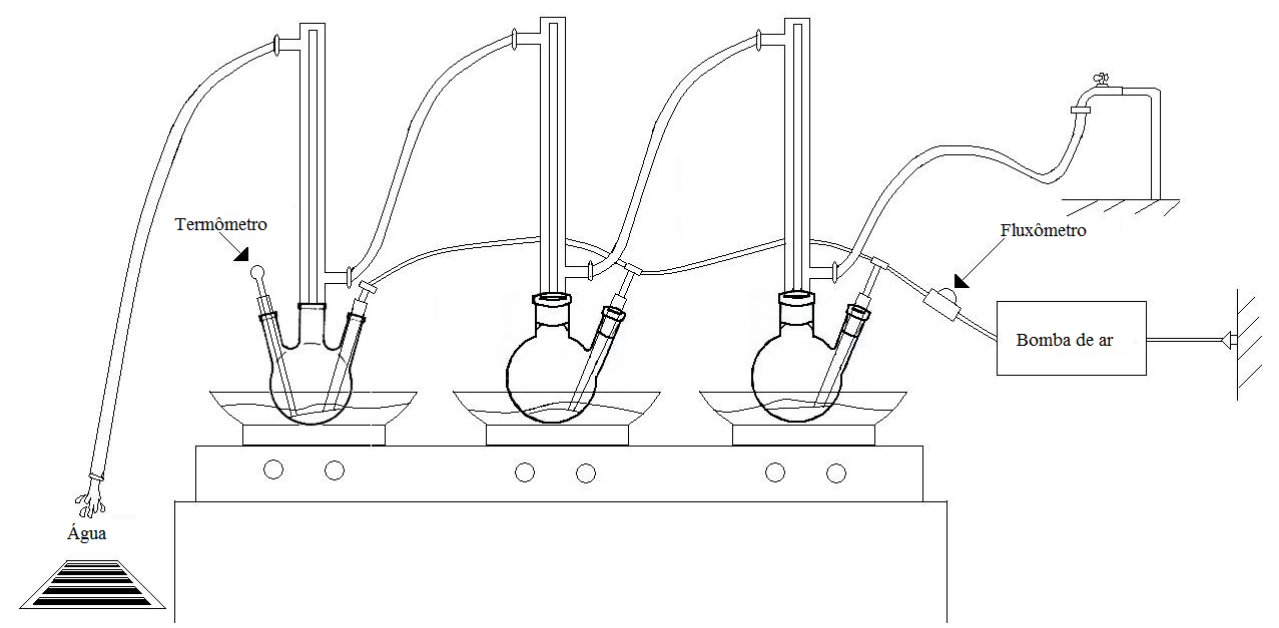

Fonte: Autoria própria. 


\subsection{Resultados e Discussão}

A espectroscopia na região do infravermelho foi empregada para monitorar a mudança nas estruturas químicas das amostras decorrentes dos processos de degradação térmica e oxidativa. O espectro na região do infravermelho do "tritolil fosfato" é mostrado na Figura 3. Pode-se observar a presença de bandas de absorção caraterísticas de fosfatos ésteres em 1586 e $1487 \mathrm{~cm}^{-1}$, sugestivas de deformação axial $\mathrm{C}=\mathrm{C}$ de anel aromático; em 1586 e $1487 \mathrm{~cm}^{-1}$, características de fosfatos ésteres; em $1296 \mathrm{~cm}^{-1}$ típica de estiramento (aromático) ${ }_{3} \mathrm{P}=\mathrm{O}$; em $1190 \mathrm{~cm}^{-1}$ sugestiva de estiramento C-O; em 1136, 1011 e $958 \mathrm{~cm}^{-1}$ de estiramento $\mathrm{P}-\mathrm{O}-\mathrm{C}_{(\text {aromático)}}$; em $779 \mathrm{~cm}^{-1}$ típica de estiramento $-\mathrm{C}-\mathrm{H}$ fora do plano e $685 \mathrm{~cm}^{-1}$ típica de deformação axial $\mathrm{C}=\mathrm{C}$ de anel aromático (Silverstein et al., 2005).

Figura 3. FTIR do tritolil fosfato antes do processo de degradação.

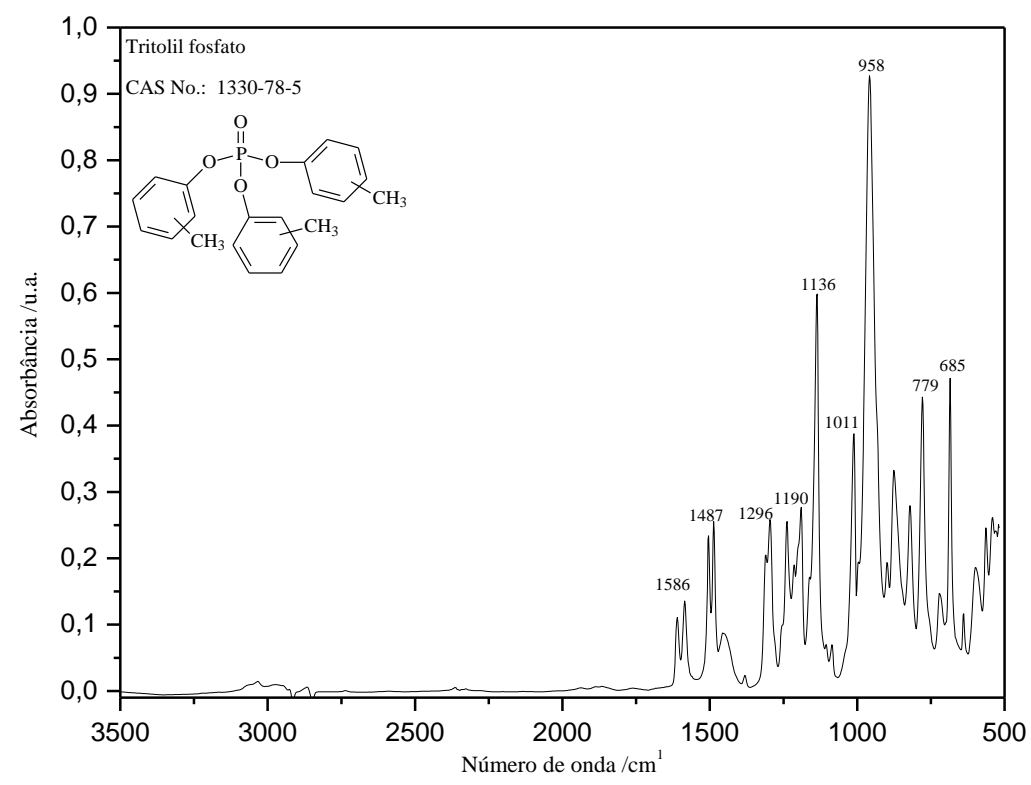

Fonte: Autoria própria.

A Figura 4 apresenta os espectros de FTIR para o composto tritolil fosfato após o processo de oxidação, com borbulhamento de ar e sem borbulhamento de ar, a uma temperatura de aquecimento de $150{ }^{\circ} \mathrm{C}\left( \pm 5^{\circ} \mathrm{C}\right)$, por 96 horas. 
Figura 4. FTIR do composto tritolil fosfato após o processo de degradação, com e sem borbulhamento de ar, a $150{ }^{\circ} \mathrm{C}\left( \pm 5^{\circ} \mathrm{C}\right)$, por 96 horas.

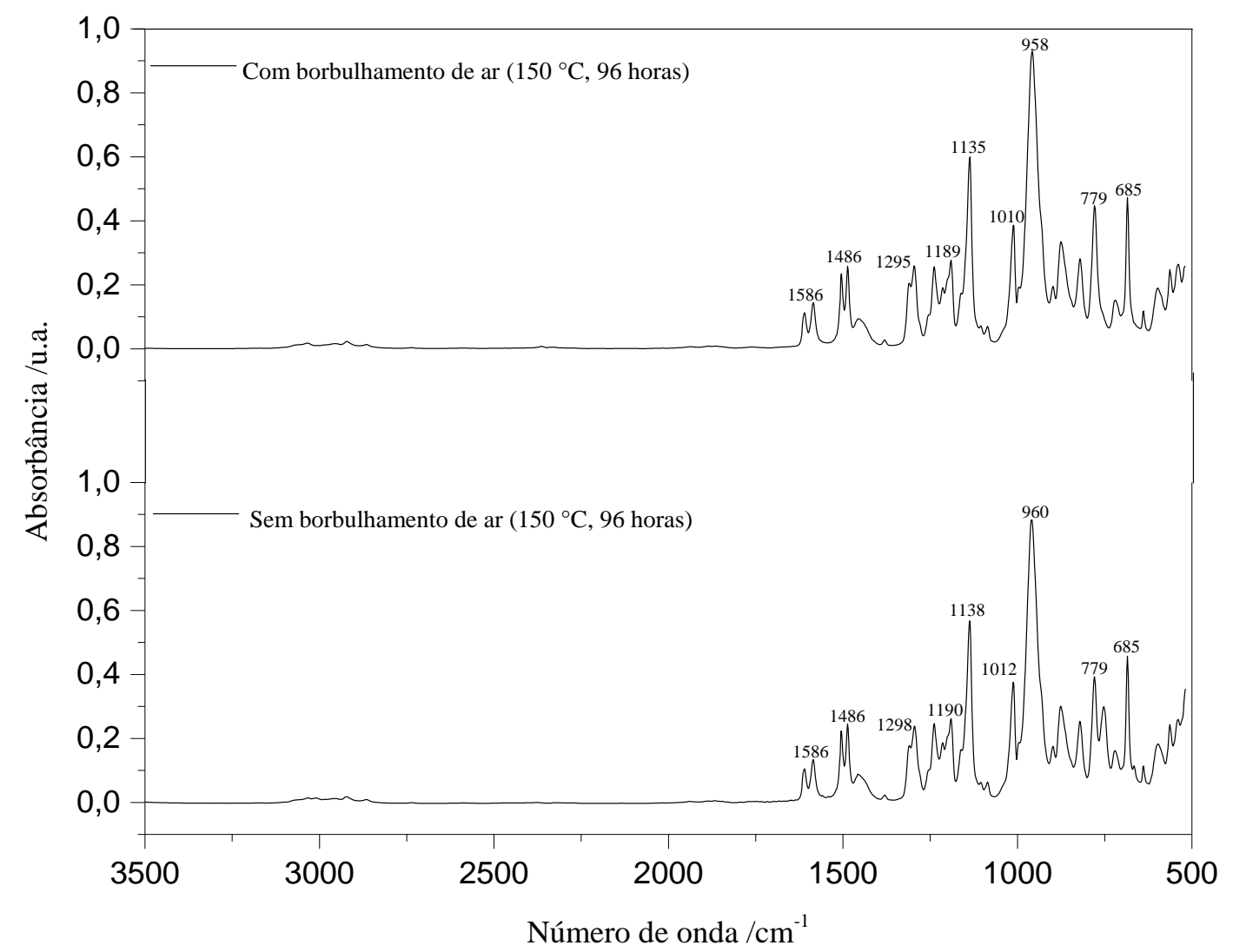

Fonte: Autoria própria.

De acordo com os resultados apresentados (ver Figuras 3 e 4), pela técnica de FTIR não foi possível observar modificações vibracionais no espectro do tritolil fosfato, para as condições de análise as quais o fosfato éster foi submetido. Entretanto, mudanças do potencial hidrogeniônico e da coloração da amostra foram observadas. Após 96 horas de degradação a amostra apresentou-se ácida e a coloração modificou de incolor para amarelo. Para ésteres de fosfato, a literatura aponta os mecanismos de degradação (pirólise, oxidação e hidrólise) levam a formação de fosfatos ácidos, ou seja, derivados do ácido fosfórico, em decorrência das condições ambientais as quais o organofosforado é submetido (presença de umidade no meio e temperaturas de moderada a alta (75 - $\left.\left.175^{\circ} \mathrm{C}\right)\right)$ (Neto et al., 2004, Pedersen e Ronningsen, 2003).

Após os testes de degradação para o tritolil fosfato, na sequência foram realizados testes de oxidação acelerada para o óleo naftênico, para verificação da estabilidade térmica e oxidativa deste derivado petroquímico. 
As Figuras 5 e 6 apresentam os espectros FTIR do óleo naftênico durante o processo de degradação térmica e oxidativa, por 168 horas.

Figura 5. FTIR do óleo naftênico durante o processo de degradação, 168 horas.

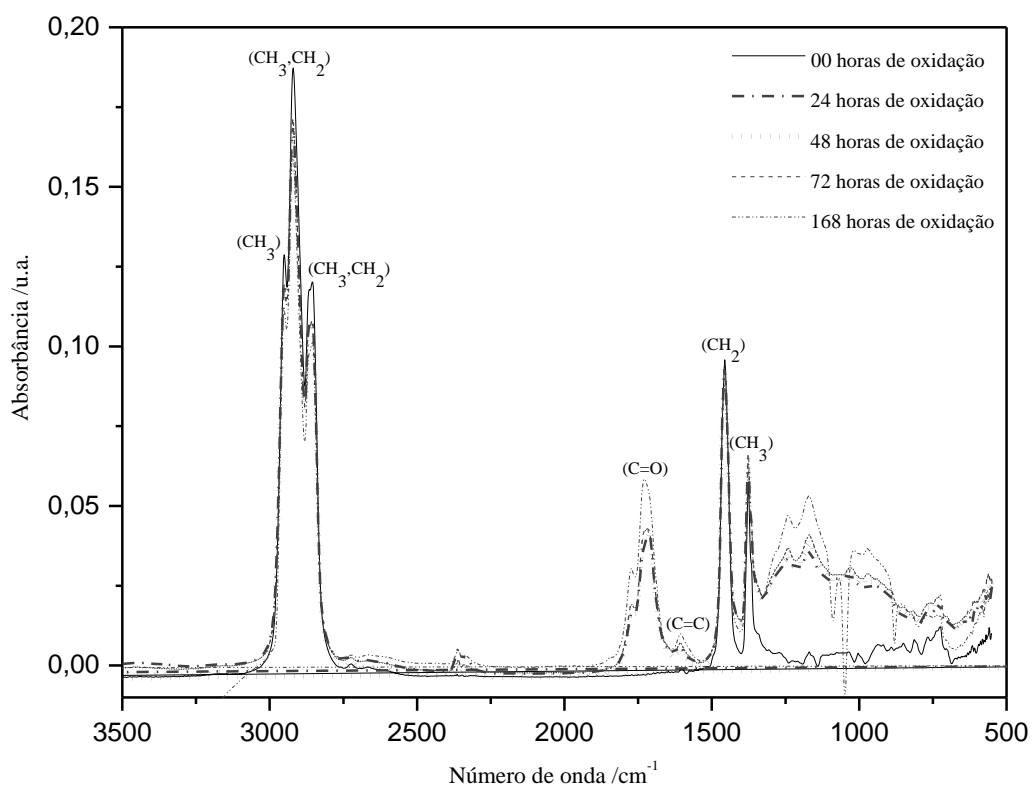

Fonte: Autoria própria.

Figura 6. FTIR do óleo naftênico durante o processo de degradação, 168 horas. Região espectral de $1900-1500 \mathrm{~cm}^{-1}$ extrapolada.

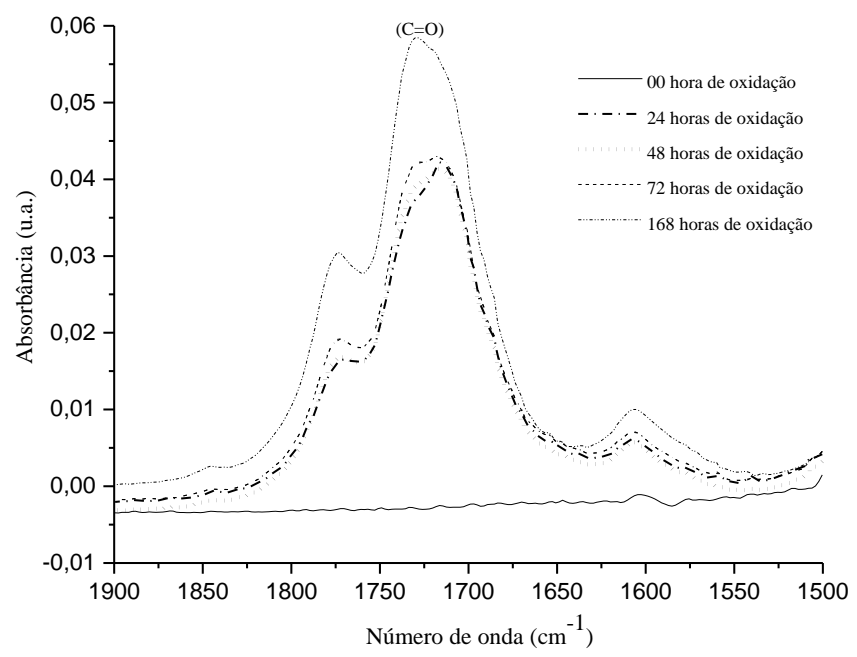

Fonte: Autoria própria 
De acordo com os resultados apresentados (ver Figuras 5 e 6) foi possível observar a vulnerabilidade do óleo naftênico à oxidação provavelmente, devido ao ataque eletrofílico do oxigênio atmosférico. Os compostos formados durante o processo oxidativo, em geral, reduzem o bom desempenho do óleo lubrificante, uma vez que ocorre alteração das propriedades físicoquímicas do referido óleo. As reações de oxidação promovem a formação de compostos carbonilados tais como, aldeídos, cetonas, peróxidos, hidroperóxidos, dentre outros, com absorções características na região entre $1700 \mathrm{~cm}^{-1}$ e $1900 \mathrm{~cm}^{-1}$ (Façanha, 2008; Rios et al., 2007). O aumento da absorção no comprimento de onda $1728 \mathrm{~cm}^{-1}(\mathrm{C}=\mathrm{O})$ caracteriza a oxidação/degradação do óleo naftênico (Silverstein et al., 2005).

\section{Análises Termogravimétricas}

Para a avaliação da estabilidade térmica e oxidativa do tritolil fosfato e do óleo naftênico foram realizadas também análises termogravimétricas em atmosfera inerte e oxidativa. As Figuras 7-10 apresentam as curvas TG-DTG para as amostras tritolil fosfato e óleo naftênico.

Figura 7. Curva termogravimétrica do tritolil fosfato em atmosfera oxidativa (ar sintético).

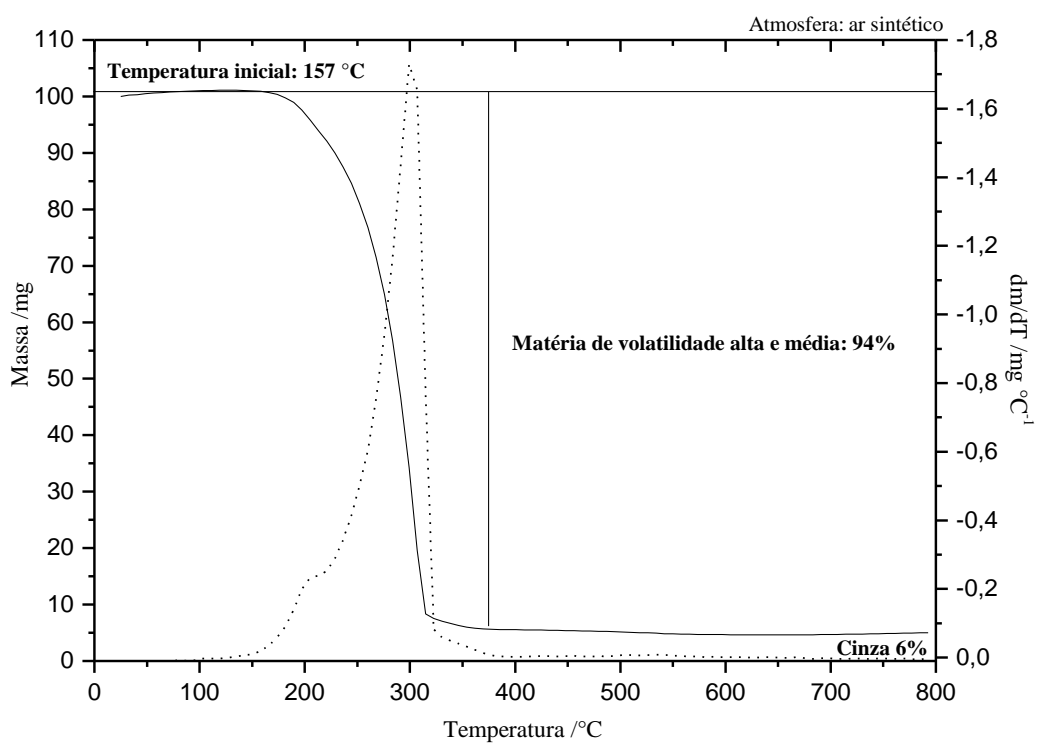

Fonte: Autoria própria. 
Figura 8. Curva termogravimétrica do tritolil fosfato em atmosfera inerte (nitrogênio).

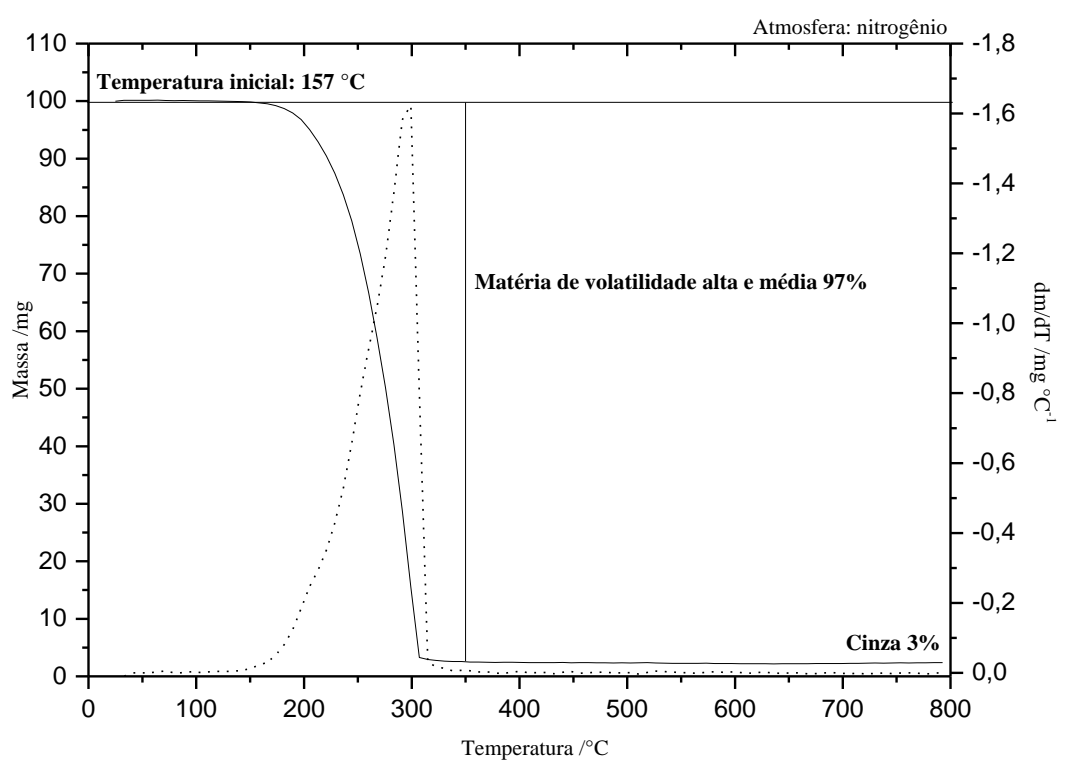

Fonte: Autoria própria.

Figura 9. Curva termogravimétrica do óleo naftênico em atmosfera oxidativa (ar sintético).

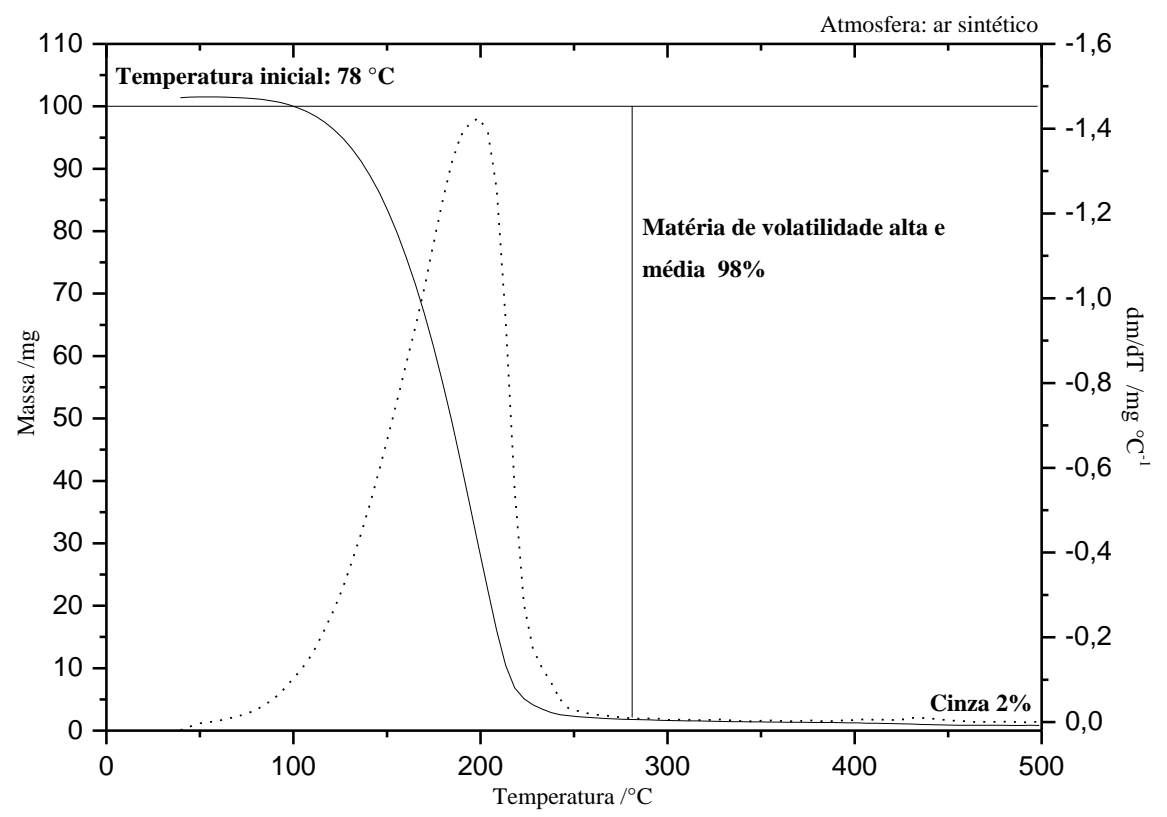

Fonte: Autoria própria. 
Figura 10. Curva termogravimétrica do óleo naftênico em atmosfera inerte (nitrogênio).

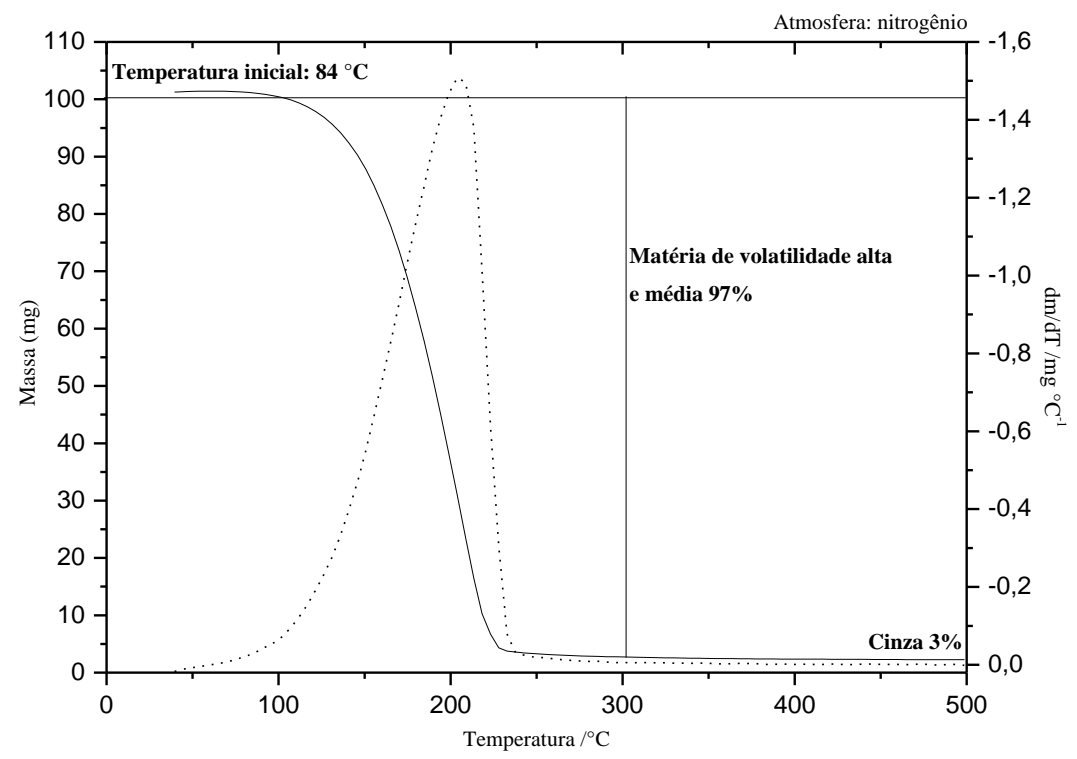

Fonte: Autoria própria.

De acordo com os resultados de análise termogravimétrica, observou-se que o tritolil fosfato possui uma boa estabilidade térmica e oxidativa, compatível com os resultados para organofosforados (Exxon Mobil Corporation, 2008). Estes compostos possuem uma boa estabilidade térmica devido à forte ligação $\mathrm{P}-\mathrm{O}-\mathrm{C}_{\text {aromático. }}$ Quanto ao óleo naftênico, o mesmo não apresentou significativa estabilidade térmica, iniciando suas etapas de oxidação/degradação em torno de 78 e $84{ }^{\circ} \mathrm{C}$ (ver Figuras 9 e 10). Os resultados de degradação obtidos por TG-DTG corroboraram com os resultados dos testes de oxidação acelerada (TOA), uma vez que o fluido hidráulico da classe dos fosfatos ésteres apresentou melhor estabilidade térmica e oxidativa quando comparado ao destilado leve de petróleo naftênico hidrotratado.

Outro dado importante extraído das curvas termogravimétricas (TG-DTG) é a determinação da temperatura máxima de decomposição. A Tabela 1 sumariza os resultados para o tritolil fosfato e para o óleo naftênico.

Tabela 1. Temperatura máxima de decomposição para as amostras.

\begin{tabular}{c|c|c}
\hline Amostra & Atmosfera & $\mathbf{T}_{\mathbf{m a x}}\left({ }^{\circ} \mathbf{C}\right)$ \\
\hline \multirow{2}{*}{ Tritolil fosfato } & Ar sintético & 300 \\
\cline { 2 - 3 } & $\mathrm{N}_{2}$ & 297 \\
\hline \multirow{2}{*}{ Óleo naftênico } & Ar sintético & 198 \\
\cline { 2 - 3 } & $\mathrm{N}_{2}$ & 206 \\
\hline
\end{tabular}

Fonte: Autoria própria. 
Os resultados de DTG também corroboraram com os dados de FTIR uma vez que o composto tritolil fosfato apresentou a maior temperatura máxima de degradação. As curvas termogravimétricas possibilitaram também o cálculo do IPDT (Integral Procedure Decomposition Temperature), o qual representa a temperatura de estabilidade térmica em que cerca de $50 \%$ do composto em análise foi degradado (Rios e Mazzetto, 2012).

Para o cálculo do IPDT foram utilizadas as áreas $S_{1}, S_{2}$ e $S_{3}$ dos termogramas (ver Figura 11), bem como as equações (1), (2) e (3) (Chiang et al., 2007; Doyle, 1961).

Figura 11. Representação das áreas do termograma para o cálculo do IPDT.

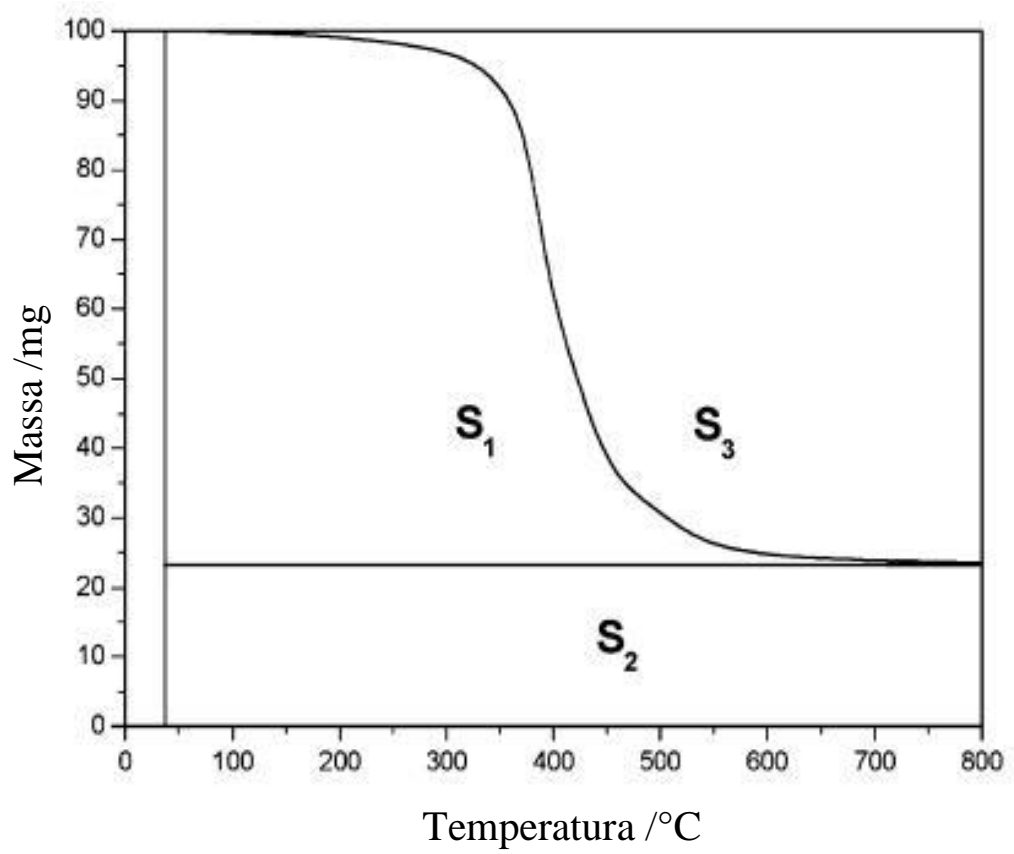

Fonte: Adaptado de Chiang et al., 2007.

Após o cálculo das áreas $\mathrm{S}_{1}, \mathrm{~S}_{2}$, e $\mathrm{S}_{3}$ utilizou-se as equações (1), (2) e (3) para a determinação do IPDT dos compostos analisados (Chiang et al., 2007).

$\operatorname{IPDT}\left({ }^{\circ} \mathrm{C}\right)=\mathrm{AK}\left(\mathrm{T}_{\mathrm{f}}-\mathrm{T}_{\mathrm{i}}\right)+\mathrm{T}_{\mathrm{i}}$

$A=\frac{S_{1}+S_{2}}{S_{1}+S_{2}+S_{3}}$

$\mathrm{K}=\frac{\mathrm{S}_{1}+\mathrm{S}_{2}}{\mathrm{~S}_{1}}$

No qual: "A" é a razão da área total da curva experimental do termograma, "K" é a razão entre a soma das áreas $S_{1}$ e $S_{2}$, pela área $S_{1}$, "T $T_{i}$ " é a temperatura inicial e " $T_{\mathrm{f}}$ ” é a temperatura final da curva termogravimétrica. A Tabela 2 apresenta os dados de IPDT. 
Tabela 2. Dados de IPDT para as amostras analisadas.

\begin{tabular}{c|c|c}
\hline Amostra & Atmosfera & IPDT $\left({ }^{\circ} \mathbf{C}\right)$ \\
\hline \multirow{2}{*}{ Tritolil fosfato } & Ar sintético & 319,4 \\
\cline { 2 - 3 } & $\mathrm{N}_{2}$ & 287,0 \\
\hline \multirow{2}{*}{ Óleo naftênico } & Ar sintético & 176,3 \\
\cline { 2 - 3 } & $\mathrm{N}_{2}$ & 186,0 \\
\hline
\end{tabular}

Fonte: Autoria própria.

Os resultados para o cálculo do IPDT também evidenciaram a boa estabilidade térmica do tritolil fosfato frente ao óleo naftênico. O fosfato éster foi a amostra que apresentou os maiores valores para o referido cálculo. Desta forma, pode-se inferir que os fosfatos ésteres são ótimas opções como fluidos hidráulicos para a indústria de máquinas pesadas uma vez que possuem elevada estabilidade térmica e oxidativa, e assim, conseguem manter suas propriedades físicoquímicas por mais tempo e consequentemente, atuam de forma eficaz por um tempo mais prolongado.

\subsection{Conclusão}

A avaliação da estabilidade térmica e oxidativa dos fluidos tritolil fosfato e óleo naftênico nos permite concluir que o fosfato éster possui uma melhor estabilidade quando comparado ao óleo naftênico. Os métodos de degradação foram eficazes no que concerne a investigação da estabilidade dos referidos compostos, quando submetidos a condições extremas de trabalho (aquecimento, umidade e presença de oxigênio). Deve-se inferir ainda que a obtenção dos parâmetros $\mathrm{T}_{\max }$ e IPDT também contribuiu sobremaneira para diagnosticar o comportamento térmico dos fluidos em questão; e os mesmos puderam contribuir para validação dos resultados obtidos a partir dos sistemas oxidativos desenvolvidos em laboratório.

\section{Agradecimentos}

Os autores agradecem aos órgãos financiadores da pesquisa: CAPES, CNPq e FAPEPI, agradecem também ao LIMAV pelas análises de FTIR e TG, bem como ao aluno Ricardo Gomes Viana (Universidade Federal do Piauí - Departamento de Química) pelos desenhos dos sistemas de oxidação. 


\section{Referências}

ALVES, W. F. Preparação e caracterização mecânica, térmica e elétrica de misturas de poliuretano derivado do óleo da mamona e poli (o-metoxianilina) para avaliação na aplicação como sensores para língua eletrônica. 2010. 125 f. Dissertação (Doutorado em ciências, área de concentração: Físico-química) - Departamento de química, Universidade Federal de São Carlos, São Carlos, 2010.

ASTM D2440-13, Standard Test Method for Oxidation Stability of Mineral Insulating Oil, 2013.

CHIANG, C. L., CHANG, R. C., CHIU, Y. C. Thermal stability and degradation kinetics of novel organic/inorganic epoxy hybrid containing nitrogen/silicon/phosphorus by sol-gel method.

Thermochimica Acta. v. 453, n. 2, pp. 97-104, 2007.

DOYLE, C. D. Estimating thermal stability of experimental polymers by empirical thermogravimetric analysis. Analytical Chemistry, v. 33, n. 1, p. 77-79, 1961.

EXXON MOBIL CORPORATION, Technical Bulletin, Stability of Phosphate Ester Aviation Hydraulic Fluids, 2008.

FAÇANHA, M. A. R., MAZZETTO, S. E.,CARIOCA, J. O. B., BARROS, G. G. Evaluation of antioxidant properties of a phosphorated cardanol compound on mineral oils (NH10 and NH20). Fuel, v. 86, p. 2416, 2007.

HABICHER, W. D., Organophosphorus antioxidants: part $X$ - mechanism of antioxidant action of aryl phosphites and phosphonites at higher temperatures, Polymer Degradation and Stability, v. 31, n. 2, p. 219-228, 1991.

HOWWEL, B. A., Al-OMARI, M. Thermal and spectroscopic characterization of triaryl phosphate hydraulic fluid. Journal of Thermal Analysis Calorimetry, v. 109; pp.1157-1161, 2012.

KIRPICHNIKOV, P.A., MUKMENEVA, N.A., POBEDIMSKII, D.G. Organophosphorus Stabilisers of Polymers: Efficiency and the Mechanisms of Action. Russian Chemical Reviews, v. 52, pp. 1051, 1983.

NETO, R.C.R., LIMA, D.O., PINHEIRO, T.D.S., ALMEIDA, R.F., DANTAS, T.N.C., DANTAS, M.S.G., ARAÚJO, M.A.S., JÚNIOR, C.L.C., AZEVEDO, D.C.S., Thermo-Oxidative Stability of Mineral Naphthenic Insulating Oils: Combined Effect of Antioxidants and Metal Passivator. Industrial \& Engineering Chemistry Research, v. 43, pp. 7428, 2004.

PEDERSEN, K., RONNINGSEN, H. P. Influence of Wax Inhibitors on Wax Appearance Temperature, Pour Point, and Viscosity of Waxy Crude Oils. Energy Fuels, v. 17, pp. 321-328, 2003.

RIOS, M. A. S., MAZZETTO, S. E. Effect of organophosphate antioxidant on the thermo-oxidative degradation of a mineral oil. Journal of Thermal Analysis and Calorimetry, v. 111, n. 1, pp. 553$559,2013$.

RIOS, M. A. S., MAZZETTO, S. E. Thermal behavior of phosphorus derivatives of hydrogenated cardanol. Fuel Processing Technology, v. 96, p.1-8, 2012.

RIOS, M. A. S., MAZZETTO, S. E., CARIOCA, J. O. B., ARRAIS, G. B. Síntese de novos aditivos oriundos de fonte renovável para aplicação na indústria de derivados do petróleo. In: $3^{\circ}$ Congresso Brasileiro de Petróleo e Gás, 2005, Salvador, BA.

RIOS, M. A. S., SANTIAGO, S. N., LOPES, A. A.S., MAZZETO, E. S. Antioxidative Activity of 5-n-Pentadecyl-2-tert-butyphenol Stabilizers in Mineral Lubricant Oil. Energy Fuels, v. 24, pp. 3285-3291, 2010. 
SANTOS, J. C. O., SANTOS, M. G., SOUZA, A. G., SOBRINHO, E. V., Jr, V. J. F., SILVA, A. J. N. Thermoanalytical and rheological characterization of automotive mineral lubricants after thermal degradation. Fuel, v. 83, pp. 2393-2399, 2004.

SCHUMACHER, R., ZINKE, H., LANDOLT, D., MATHIEU, H. J. Improvement of lubrication breakdown behaviour of isogeometrical phosphorus by antioxidants. Wear, v. 146, pp. 25-35, 1991.

SHUMACHER, R., ZINKE, H. Tribofragmentation and antiwear behavior of isogeometric phosphorus compounds. Tribology International, v. 30, n. 3, pp. 199-208, 1997.

SILVERSTEIN, R.M., WEBSTER, F.X., KIEMLE, D.J., Spectrometric Identification of Organic Compounds., 7th ed., John Wiley \& Sons, New York, (2005).

Recebido: 15/12/2014

Aprovado: 29/05/2015 